Pacific

Journal of

Mathematics

ARF INVARIANTS OF REAL ALGEBRAIC CURVES

PATRICK M. GILMER

Volume $230 \quad$ No. 2

April 2007 


\title{
ARF INVARIANTS OF REAL ALGEBRAIC CURVES
}

\author{
PATRICK M. GILMER \\ Dedicated to the memory of my adviser, P. Emery Thomas
}

\begin{abstract}
We give new congruences for singular real algebraic curves, generalizing Fiedler's congruence for nonsingular curves.
\end{abstract}

\section{Introduction}

Let $A_{\mathbb{R}}$ be an irreducible real algebraic curve of degree $2 k$ with only real nodal singularities. $A_{\mathbb{R}}$ consists of the image of a number of immersed circles. $A_{\mathbb{R}}$ is called an M-curve if the number of immersed circles plus the number of double points is $1+\left(\begin{array}{c}2 k-1 \\ 2\end{array}\right)$. Let $A_{\mathbb{C}}$ be the complex curve in $\mathbb{C P}(2)$ given by the same polynomial as $A_{\mathbb{R}}$. Thus $A_{\mathbb{R}}=A_{\mathbb{C}} \cap \mathbb{R P}(2) . A_{\mathbb{R}}$ is an M-curve precisely when $A_{\mathbb{C}} \backslash A_{\mathbb{R}}$ consists of two punctured spheres interchanged by complex conjugation. Arbitrarily choose one of these components, say $A_{\mathbb{C}}{ }^{+}$. The complex structure on $A_{\mathbb{C}}{ }^{+}$induces an orientation on $A_{\mathbb{C}}{ }^{+}$, and thus on each immersed circle of $A_{\mathbb{R}}$. Of course if we choose the other component, we get the opposite orientation on each immersed circle of $A_{\mathbb{R}}$. An orientation on each of the components up to reversing all the orientations simultaneously is called a semiorientation. Thus each M-curve receives a semiorientation, called the complex orientation [Rokhlin 1978].

An oval is a two-sided simple closed curve in the real projective plane $\mathbb{R P}(2)$. The inside of an oval is the component of its complement that is a disk; the outside is a Möbius band. Suppose $\mathscr{C}$ is a simple curve, that is, a disjoint collection of oriented ovals. An oval of $\mathscr{b}$ is called even or odd according to whether it lies inside an even or odd number of other ovals of $\mathscr{C}$. Let $p(\mathscr{C})$ denote the number of even ovals in $\mathscr{C}$, and $n(\mathscr{C})$ the number of odd ovals in $\mathscr{C}$. If one of ovals of $\mathscr{C}$ lies inside another oval of $\mathscr{C}$ or vice versa, we say they are linked. We say $\mathscr{C}$ is odd if each oval is linked with an odd number of other ovals; thus an odd curve must have an even number of components. We say $\mathscr{C}$ is even if each oval is linked with an even number of other ovals and the total number of ovals is odd.

MSC2000: primary 14P25; secondary 57M27.

Keywords: nodal curve, oval, link. 
We denote by $\Pi^{+}(\mathscr{C})$ the number of pairs of linked ovals for which the orientations on the curves extend to an orientation of the intervening annulus, and by $\Pi^{-}(\mathfrak{b})$ the number of pairs of linked ovals for which this is not the case.

We will usually write simply $n, p, \Pi^{ \pm}$without $\mathscr{C}$, except in ambiguous instances.

By a curve we will mean a collection of immersed oriented curves in $\mathbb{R P}(2)$ with only transverse double point intersections. We say two curves $A$ and $A^{\prime}$ are weakly equivalent if they can be connected via a sequence of ambient isotopies in $\mathbb{R P}(2)$, plus local moves and their inverses: balanced type I moves (see figure), safe type II moves, type III moves, and "empty" figure-eight deaths. ${ }^{1}$

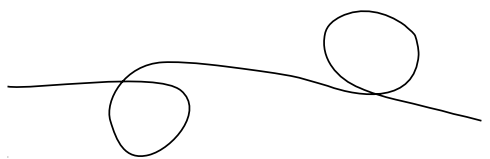

A balanced type I move replaces an arc having two curls, as shown, by an arc without double points.

Theorem 1.1. Let $A_{\mathbb{R}}$ be a nodal $M$-curve of degree $2 k$.

If $k$ is even and $A_{\mathbb{B}}$ is weakly equivalent to an odd simple curve $\mathscr{C}$, then

$$
\Pi^{+}(\mathfrak{b})-\Pi^{-}(\mathfrak{b})-p(\mathscr{C}) \equiv \frac{1}{2} k^{2} \text { or }\left(\frac{1}{2} k^{2}-2\right)(\bmod 8) .
$$

If $k$ is odd and $A_{\mathbb{R}}$ is weakly equivalent to an even simple curve $\mathscr{C}$, then

$$
\Pi^{+}(\mathfrak{b})-\Pi^{-}(\mathscr{C})-n(\mathscr{C}) \equiv \frac{1}{2}\left(k^{2}-1\right)(\bmod 8) .
$$

In the case $A_{\mathbb{R}}=\mathscr{C}$, this reduces to a congruence due to Fiedler [1983]. The reduction is not obvious; we prove it explicitly in Theorem 5.4. Fiedler [1986] has also given generalizations of his congruence to singular curves. The scheme

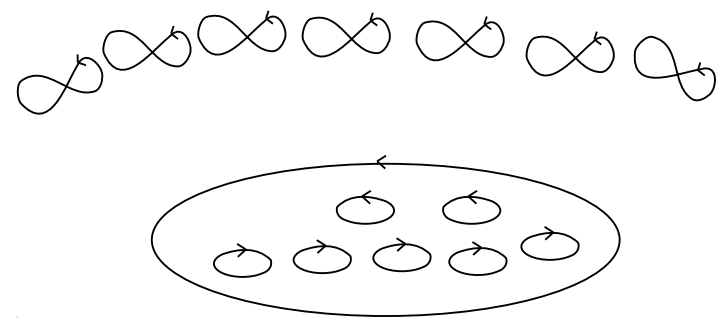

for a degree- 8 nodal M-curve with complex orientation is prohibited by Theorem 1.1 but not by the results of [Fiedler 1986]. Nor is it prohibited by any of the

\footnotetext{
${ }^{1}$ Type II and III moves mean Reidemeister moves of these types on diagrams without over- or undercrossings. In the type II case, "safe" means we require in addition that the two strands have opposite orientations.
} 
other known general restrictions on nodal curves: the Kharlamov-Viro congruences [1988] (as correctly stated in [Viro and Orevkov 2001]), the Viro inequalities [Viro 1978; Finashin 1996; Gilmer 2000], or the extremal properties of the Viro inequalities in [Gilmer 2000]. If we perturb the figure-eights in this scheme into pairs of ovals we obtain the scheme for a nonsingular M-curve $\langle 14 \coprod 1\langle 7\rangle\rangle$ which can be realized by a real algebraic curve [Viro 1986].

Theorem 1.1 is a corollary of Theorem 5.3 below, which in turn is a simplified version of the more general Theorem 3.3 of [Gilmer 2000]. To prove (or even state) Theorem 5.3, we must discuss the Arf invariant of links, which we do in Sections 2 and 3. These sections are, mainly, a review of parts of [Gilmer 1993a; 1993b].

In Section 6 we show that the two explicit examples given by Fiedler of curves prohibited by his congruence [1986] for singular curves are prohibited by our Theorem 5.3 as well. It seems likely that any scheme for a curve with complex orientation that can be prohibited by Fiedler's congruence can also be prohibited by Theorem 5.3. However our theorem applies to hypothetical curves with a complex orientation. Fiedler's result concerns hypothetical singular curves which, by hypothesis, are related to actual nonsingular real algebraic curves by desingularization. Thus information about the complex orientations of the hypothetical curves is contained only implicitly in the relation to the actual nonsingular real algebraic curve. For this reason, it seems difficult to derive [Fiedler 1986] as a corollary of Theorem 5.3 in a way similar to the proof of Theorem 5.4. However both obstructions can be interpreted as deriving from the calculation of the Brown invariant of the Gillou-Marin form on characteristic surfaces. Moreover the two surfaces are closely related.

\section{Arf invariants of links in $S^{3}$}

An oriented link in $S^{3}$ is called proper if for each component the sum of the linking numbers with all the other components is even. That is, $L=\sqcup_{i} K_{i}$ is proper if and only if $\operatorname{lk}\left(K_{i}, L-K_{i}\right) \equiv 0(\bmod 2)$ for all $i$. We use $1 \mathrm{k}$ to denote the $\mathbb{Z}$-valued linking number of oriented links.

Robertello defined the Arf invariants of proper links and gave several equivalent definitions. One involved the Seifert pairing on an orientable spanning surface. We generalized this definition so that it applies to nonorientable spanning surfaces [Gilmer 1992; 1993a]. This definition for the Arf invariant is analogous to the Gordon-Litherland [Gordon and Litherland 1978] definition of the signature of a knot. There is a version for unoriented links, but the oriented link version is more useful in this paper.

Let $V$ be a $\mathbb{Z} / 2 \mathbb{Z}$ vector space equipped with a symmetric bilinear form

$$
\cdot: V \times V \rightarrow \mathbb{Z} / 2 \mathbb{Z}
$$


A function $q: V \rightarrow \mathbb{Z} / 4 \mathbb{Z}$ is called a quadratic refinement of $\cdot$ if

$$
q(x+y)-q(x)-q(y)=2 x \cdot y
$$

for all $x, y \in V$. Here 2 denotes the nontrivial group homomorphism $\mathbb{Z} / 2 \mathbb{Z} \rightarrow$ $\mathbb{Z} / 4 \mathbb{Z}$. Let rad be the radical of $\cdot$. We say that $q$ is proper if $q$ vanishes on $\operatorname{rad}$. If $q$ is proper, the Brown invariant $\beta(q) \in \mathbb{Z} / 8 \mathbb{Z}$ is defined by the equation

$$
e^{2 \pi i \beta(q) / 8}=\frac{1}{(\sqrt{2})^{\operatorname{dim} V+\operatorname{dim} \operatorname{rad}}} \sum_{v \in V} i^{q(v)} .
$$

The Brown invariant is additive for the direct sum of quadratic refinements. A simple graphical scheme for writing a given form as a direct sum of simple elementary forms and thus calculating the Brown invariant is given in [Gilmer 1993a; 1993b].

Let $L$ be a link in $S^{3}$ with oriented components $\left\{K_{i}\right\}$. Let $F$ be a not necessarily orientable spanning surface for $L$. Define a map

$$
q_{F}: H_{1}(F, \mathbb{Z} / 2 \mathbb{Z}) \rightarrow \mathbb{Z} / 4 \mathbb{Z}
$$

as follows. Given $x \in H_{1}(F, \mathbb{Z} / 2 \mathbb{Z})$, pick a simple closed curve $\alpha_{x}$ representing $x$, and define $q_{F}(x)$ to be the number of positive half twists in a tubular neighborhood of $x$ in $F$. More precisely,

$$
q_{F}(x)=\operatorname{lk}\left(\alpha_{x}, \hat{\alpha}_{x}\right) .
$$

Here $\hat{\alpha}_{x}$ is the boundary of a tubular neighborhood of $\alpha_{x}$ oriented in the same direction as some arbitrarily chosen orientation for $x$. The function $q_{F}$ is well defined and is a quadratic refinement of the intersection pairing

$$
\cdot: H_{1}(F, \mathbb{Z} / 2 \mathbb{Z}) \times H_{1}(F, \mathbb{Z} / 2 \mathbb{Z}) \rightarrow \mathbb{Z} / 2 \mathbb{Z} .
$$

We note that

$$
q_{F}\left(\left[K_{i}\right]\right)=0 \Longleftrightarrow \operatorname{lk}\left(K_{i}, L-K_{i}\right) \equiv 0(\bmod 2) .
$$

Let $\bar{F}$ denote the quotient space of $F$ obtained by identifying each component of $F$ to a point corresponding to that component. Let $\pi: F \rightarrow \bar{F}$ denote the quotient map. Thus $L$ is proper if and only if $q_{F}\left[K_{i}\right]=0$ for all $i$ if and only if $q_{F}$ can be factored through $\pi_{*}$.

Define

$$
\mu(F)=\frac{1}{2} \sum_{i, j} 1 \mathrm{k}\left(K_{i}, K_{j}^{\prime}\right),
$$

where $K_{j}^{\prime}$ denotes $K_{j}$ pushed slightly into the interior of $F$. Then set

$$
\operatorname{Arf} L=\beta\left(q_{F}\right)-\mu(F) \in \mathbb{Z} / 8 \mathbb{Z} \text {. }
$$


One can relate any two spanning surfaces by a sequence of moves of certain types (and their inverses):

(0) isotopy;

(1) adding a hollow handle;

(2) replacing a collar of the boundary with a punctured Möbius band, obtained by adding an unknotted positively or negatively half- twisted band (see Figure 7 in [Gordon and Litherland 1978]);

(3) the birth of an empty two-sphere in a small 3-ball, as described in [Gilmer 1993a].

The equivalence relation generated by these moves is called $S^{*}$-equivalence. Since $\beta\left(q_{F}\right)-\mu(F)$ is preserved by these moves, $\operatorname{Arf} L$ is well defined. It takes values in $4 \mathbb{Z} / 8 \mathbb{Z}$. It is invariant under oriented band summing, and adding and removing small unlinked unknots. This implies that it is an invariant of planar cobordism [Gilmer 1993a].

Consider the trefoil below, which is spanned by a Möbius band $F$. The group $H_{1}(F, \mathbb{Z} / 2 \mathbb{Z}) \approx \mathbb{Z} / 2 \mathbb{Z}$ is generated by the core $x$. We have $q_{F}(x) \equiv-3 \equiv 1(\bmod 4)$, $\beta\left(q_{F}\right) \equiv 1(\bmod 8), \mu=\frac{-6}{2}=-3$, and Arf(trefoil) $\equiv 4(\bmod 8)$.

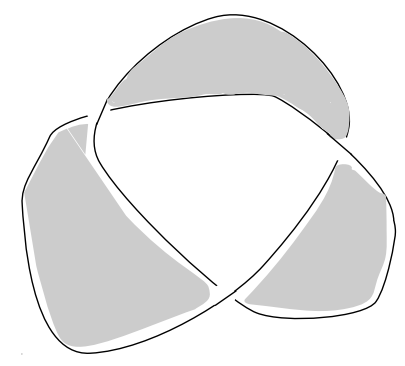

\section{Arf invariants of links in rational homology spheres}

Let $L$ be a link in a rational homology sphere $M$ with oriented components $\left\{K_{i}\right\}$. By a spanning surface for $L$ we mean a possibly nonorientable surface $F$ in $M$ with boundary $L$. We can speak of $S^{*}$-equivalence of spanning surfaces in $M$. Two spanning surfaces for a link need not be $S^{*}$-equivalent. We introduced a parameter to index $S^{*}$ equivalence classes of spanning surfaces in [Gilmer 1993a]. Suppose the homology class of $L$ represents zero in $H_{1}(M, \mathbb{Z} / 2 \mathbb{Z})$. Then

$$
\Gamma(L)=\left\{\gamma \in H_{1}(M) \mid 2 \gamma=[L] \in H_{1}(M)\right\}
$$

is nonempty.

Let $F$ be a spanning surface for $L$ with no closed components, and $i_{F}: F \rightarrow M$ be the inclusion. $H_{1}(F)$ is free abelian and the homology class of $L$ the boundary 
of $F$ equipped with the string orientation of the link represents a homology class in $H_{1}(F)$ which is divisible by two. Define

$$
\gamma(F)=i_{F}\left(\frac{1}{2}[L] \in H_{1}(F)\right) \in \Gamma(L) \subset H_{1}(M) .
$$

If $F$ has some closed components, we take $\gamma(F)$ to be $\gamma\left(F^{\prime}\right)$, where $F^{\prime}$ is formed by deleting the interior of a disk from each closed component and orienting the new boundary components in an arbitrary way. For further discussion, see [Gilmer 1992].

Then $\gamma(F)$ is preserved by $S^{*}$-equivalence, and the map from $S^{*}$-equivalence classes of spanning surfaces for $L$ to $\Gamma(L)$ is bijective. By abuse of notation, we let $F_{\gamma}$ denote a spanning surface $F$ with $\gamma\left(F_{\gamma}\right)=\gamma$. This should not cause any confusion.

The number of positive half twists in a tubular neighborhood of a curve on a surface in $M$ has no well-defined analog in this more general situation. What we actually need is an analog of the number of half twists modulo four.

We do have a linking number in $\mathbb{Q}$ which can be defined for disjoint 1-cycles. We continue to denote this linking number by $\mathrm{lk}$. We do know what it means to increase or decrease the number of half twists in the neighborhood of a curve $\alpha$. Moreover if we add a half twist to a neighborhood of $\alpha$, we increase the linking number of $\alpha$ and the boundary of the neighborhood of $\alpha$ by one.

But we do not know what untwisted (mod 4) should mean. As a replacement for this, it suffices to fix a quadratic refinement of the linking form of $M$ [Gilmer 1993b]. The linking form of $M$,

$$
\ell_{M}: H_{1}(M) \times H_{1}(M) \rightarrow \mathbb{Q} / \mathbb{Z},
$$

is a bilinear form with an injective adjoint. A quadratic refinement of $\ell_{M}$ is a function

$$
r: H_{1}(M) \rightarrow \mathbb{Q} / \mathbb{Z}
$$

such that

$$
r(x+y)-r(x)-r(y) \equiv \ell_{M}(x, y)(\bmod 1) .
$$

It follows that the boundary $\hat{\alpha}$ of a neighborhood of an oriented curve $\alpha$ on a surface $F$ will have $\operatorname{lk}(\hat{\alpha}, \alpha) \equiv r(3[\alpha])-r(2[\alpha])-r([\alpha]) \equiv\left(3^{3}-2^{2}-1\right) r([\alpha]) \equiv$ $4 r([\alpha])(\bmod 1)$. Thus we may define

$$
q_{r, F}: H_{1}(F, \mathbb{Z} / 2 \mathbb{Z}) \rightarrow \mathbb{Z} / 4 \mathbb{Z}
$$

by

$$
q_{r, F}(x) \equiv \operatorname{lk}\left(\gamma_{x}, \hat{\gamma}_{x}\right)-4 r(x)(\bmod 4) .
$$

This is a quadratic refinement of the intersection form on $H_{1}(F, \mathbb{Z} / 2 \mathbb{Z})$, and is well defined by [Gilmer 1993a, Theorem 6.1]. 
We also have, by [Gilmer 1993a, Proposition 6.3],

$q_{r, F}\left(\left[K_{i}\right]\right)=0 \Longleftrightarrow \operatorname{lk}\left(K_{i}, L-K_{i}\right) \equiv 2 \ell_{M}\left(\left[K_{i}\right], \gamma(F)\right)-2 r\left(\left[K_{i}\right]\right)(\bmod 2)$.

Note that $q_{r, F_{\gamma}}\left[K_{i}\right]=0$ for all $i$ if and only if $q_{r, F_{\gamma}}$ can be factored through $\pi_{*}$. We say $(L, \gamma, r)$ is proper if either of these equivalent conditions holds, that is, if

$$
\operatorname{lk}\left(K_{i}, L-K_{i}\right) \equiv 2 \ell_{M}\left(\left[K_{i}\right], \gamma(F)\right)-2 r\left(\left[K_{i}\right]\right)(\bmod 2) \text { for all } i .
$$

As in the previous section, we define

$$
\mu\left(F_{\gamma}\right)=\frac{1}{2} \sum_{i, j} \operatorname{lk}\left(K_{i}, K_{j}^{\prime}\right)
$$

where $K_{j}^{\prime}$ denotes $K_{j}$ pushed slightly into the interior of $F_{\gamma}$.

If $(L, \gamma, r)$ is proper, we define

$$
\operatorname{Arf}(L, \gamma, r)=\beta\left(q_{r, F_{\gamma}}\right)-\mu\left(F_{\gamma}\right) \in \mathbb{Q} / 8 \mathbb{Z} .
$$

As for proper links in $S^{3}$, Arf is well defined and is an invariant of planar cobordism. It is also invariant under oriented band summing, and adding and removing small unlinked unknots. When defined, $\operatorname{Arf}(L, \gamma, r)$, taken modulo four, depends only on $\gamma$ and $r$ [Gilmer 1993a, Proposition 6.9].

(In [Gilmer 1993a; 1996] our $\mu$ above is broken up into the sum of two terms, $\lambda(L)=\sum_{i<j} \operatorname{lk}\left(K_{i}, K_{j}\right)$ and $-\frac{1}{2} e\left(F_{\gamma}\right)=\frac{1}{2} \sum_{i} \operatorname{lk}\left(K_{i}, K_{i}^{\prime}\right)$. For our purposes in this paper, the use of $\mu$ makes things simpler.) We note that

$$
\mu\left(F_{\gamma}\right) \equiv \ell([L], \gamma)(\bmod 1) .
$$

This restricts the range of values $\operatorname{Arf}(L, \gamma, r)$ can take.

Both $\Gamma(L)$ and the set of all quadratic refinements of $\ell(M)$ are free $H^{1}\left(M, \mathbb{Z}_{2}\right)$ sets. Properness of links is preserved under the action which changes both $\gamma$ and $r$ simultaneously by [Gilmer 1996, 6.5]. Moreover $\operatorname{Arf}(L, \gamma, r)$ changes in a nice way under this action

Proposition 3.1 [Gilmer 1996, 6.5]. If $(L, \gamma, q)$ is proper then $(L, \psi \cdot \gamma, \psi \cdot q)$ is proper and

$$
\operatorname{Arf}(L, \psi \cdot \gamma, \psi \cdot q)=\operatorname{Arf}(L, \gamma, q)+\operatorname{Arf}(\varnothing, \psi \cdot 0, \psi \cdot q)
$$

\section{The tangent circle bundle of the real projective plane}

We will be concerned with links in the tangent circle bundle of $\mathbb{R P}(2)$ which we will denote $\mathscr{T}$. This is the boundary of $\mathscr{D}$, the tangent disk bundle of $\mathbb{R P}(2)$. $\mathscr{D}$, of course, has the homology of $\mathbb{R P}(2)$. It follows that $\mathscr{T}$ is a rational homology sphere. In fact $\mathscr{T}$ is the lens space $L(4,3)$ [Gilmer 1992]. We let $\ell$ denote $\ell_{\mathscr{T}}$. 
By an $\mathscr{I}$-curve, we mean a collections of immersed oriented curves in $\mathbb{R P}(2)$ where each curve is never tangent to itself or any other curve in the collection. We can describe links in $\mathscr{T}$ by $\mathscr{I}$-curves. Lying over an $\mathscr{I}$-curve $C$ in $\mathscr{T}$, we have a link $\mathscr{T}(C)$ consisting of all the rays tangent to a part of $C$ and pointing in the direction of the orientation. We let $g$ denote the homology class of $\mathcal{T}$ represented by the oriented knot in $\mathscr{T}$ which lies over a straight line with either orientation. (There is an isotopy that can be obtained by spinning the line around a point on the line. It sends a line in $\mathbb{R P}(2)$ with one orientation to the same line with the opposite orientation.) The homology class $g$ is a generator for $H_{1}(\mathscr{T})$. An oval represents $2 g$.

Whenever we have an invariant of links in $\mathscr{T}$, we obtain an invariant of $\mathscr{T}$-curves. We use the same symbol to denote an invariant of oriented links in $\mathscr{T}$ and the corresponding invariant of $\mathscr{\Phi}$-curves. An immersed circle in an $\mathscr{\Phi}$-curve $C$ is called a component of $C$, and describes a component of the link $\mathscr{T}(C)$. This should not cause any confusion and simplifies our expressions.

Similarly, if we have two $\Phi_{\text {-curves }} C_{1}$ and $C_{2}$ which are never tangent to each other, we can speak of their linking number $\operatorname{lk}\left(C_{1}, C_{2}\right) \in \frac{1}{4} \mathbb{Z}$. In [Gilmer 1992], we worked out the linking numbers of some two component $\mathscr{I}$-curves. Two linked ovals (see Section 1) oriented in the same direction (so they contribute to $\Pi^{-}$) have linking number 1. Two linked ovals that are oriented oppositely (so they contribute to $\Pi^{+}$) have linking number -1 . Two unlinked ovals have linking number zero.

A one-sided simple closed curve and a disjoint oval have linking number $\frac{1}{2}$, if the oval is homologous to twice the one-sided curve in the Möbius band formed by deleting the interior of the oval from $\mathbb{R P}(2)$. Otherwise the linking number is $-\frac{1}{2}$. A one-sided simple closed curve and oval which the curve meets twice transversally have linking number $-\frac{1}{2}$. Two one-sided simple closed curves which meet in one point have linking number $-\frac{1}{4}$. Thus $\ell(g, g)=-\frac{1}{4}$, and there are two quadratic refinements of $\ell, r_{-1 / 8}$ with $r_{-1 / 8}(g)=\frac{-1}{8}$, and, $r_{3 / 8}$ with $r_{3 / 8}(g)=\frac{3}{8}$.

A dangerous type II move (that is, a nonsafe type II Reidemeister move on diagrams) between two components which reduces the number of double points by two leads to a new $\mathscr{I}$-curve where the linking number between the two components has been increased by one. Smoothing (according to the orientations) a double point of an $\mathscr{I}$-curve is called a smoothing move. A smoothing move corresponds to a oriented band move to the corresponding link in $\mathcal{T}$. Thus if we wish to calculate a linking number between two sub- $\mathscr{S}$-curves of an $\mathscr{I}$-curve, we can smooth the double points of sub- $\mathscr{S}$-curves without changing the relevant linking numbers. Of course the reverse of this smoothing move which we call an unsmoothing move will introduce a double point and also corresponds to an oriented band move performed on the corresponding link in $\mathscr{T}$.

Lying above an empty figure-eight curve is a local unknot in $\mathscr{T}$. The easiest way to see this is to note that any empty figure-eight is isotopic to an $\mathscr{I}$-curve 
which does not use every tangent direction as one travels around a circuit. Thus this $\mathscr{I}$-curve lies in an interval bundle over a disk in $\mathbb{R P}(2)$ which includes the figure-eight. Moreover the $\mathscr{I}$-curve projects to a curve in this disk with only one double point. Also, lying above two $\mathscr{I}$-curves related by balanced type I move, a safe type II or a type III move are isotopic links in $\mathscr{T}$. Thus weakly equivalent $\Phi$-curves are proper for that same $\gamma$ and $r$, and have the same Arf invariant, when it is defined.

As an example, consider the $\mathscr{I}$-curve, called td given by the diagram for trefoil in Figure 2, with the three crossings (made double points) placed in an affine part of $\mathbb{R P}(2)$. A Möbius band $F$ is described by a "vector field" ${ }^{2}$ on the shaded region which extends the tangential field on the boundary. Let $\delta$ denote a 1-sided curve in $F$ which represents the homology class $2 g$. Thus $\gamma(F)=2 g$. The linking number of the boundary of $F$ with a parallel may be calculated using the above techniques. It is -2 . Thus $\mu(F)=-1$. Moreover it follows that

$$
q_{-1 / 8, F}(\delta)=q_{3 / 8, F}(\delta)=1 .
$$

Thus Arf(td, $\left.2 g, \frac{-1}{8}\right) \equiv \operatorname{Arf}\left(\mathrm{td}, 2 g, \frac{3}{8}\right) \equiv 2(\bmod 8) . \mathrm{td}$ is actually a nodal M-curve of degree 4. This is a small confirmation of Theorem 5.3 below.

\section{Proposition 4.1.}

$\operatorname{Arf}\left(\varnothing, 2 g, r_{-1 / 8}\right) \equiv 2(\bmod 8) \quad$ and $\operatorname{Arf}\left(\varnothing, 2 g, r_{3 / 8}\right) \equiv-2(\bmod 8)$.

If either $\left(L, \gamma, r_{-1 / 8}\right)$ or $\left(L, \gamma+2 g, r_{3 / 8}\right)$ is proper for some $\gamma$, then the other is proper, and

$$
\operatorname{Arf}\left(L, \gamma, r_{-\frac{1}{8}}\right)-\operatorname{Arf}\left(L, \gamma+2 g, r_{3 / 8}\right) \equiv 2(\bmod 8) .
$$

Proof. Fix a line in $\mathbb{R P}(2)$ and consider the Klein bottle $K$ in $\mathcal{T}$ given by the set of all directions through points on this line. $K$ is a spanning surface for the empty link. The lift of this line with one orientation $\alpha$, and the lift $\alpha^{\prime}$ with the other orientation each have neighborhoods which are Möbius bands. These neighborhoods may be isotoped off of $K$ so that they are the two lifts to $\mathscr{T}$ of the Möbius bands neighborhood of the line in $\mathbb{R P}(2)$. Since $\operatorname{lk}(\alpha, \hat{\alpha})$ and $\operatorname{lk}\left(\alpha^{\prime}, \hat{\alpha}^{\prime}\right)$ are seen to be $\frac{1}{2}$, we calculate that

$$
\begin{aligned}
q_{r_{-1 / 8}, K}(\alpha) & =q_{r_{-1 / 8}, K}\left(\alpha^{\prime}\right)=\frac{1}{2}-4 \cdot \frac{-1}{8}=1, \\
q_{r_{3 / 8}, K}(\alpha) & =q_{r_{3 / 8}, K}\left(\alpha^{\prime}\right)=\frac{1}{2}-4 \cdot \frac{3}{8}=-1 .
\end{aligned}
$$

In this way we obtain the first two equations. The rest follows from Proposition 3.1 .

\footnotetext{
${ }^{2}$ At the double points, we have a whole arc of lines joining the two intersecting lines which fill out the shaded region.
} 
The following is a special case of [Gilmer 2000, Proposition 3.2]. Its proof is a simple calculation using as a spanning surface the union of $k$ disjoint annuli swept out by rotating $k$ lines.

Proposition 4.2. Let $X_{2 k}$ be $2 k$ lines in general position. Then $\left(X_{2 k}, k g, r_{-1 / 8}\right)$ and $\left(X_{2 k},(k+2) g, r_{3 / 8}\right)$ are proper, and

$$
\operatorname{Arf}\left(X_{2 k}, k g, r_{-1 / 8}\right) \equiv \frac{1}{2} k^{2} \equiv \operatorname{Arf}\left(X_{2 k},(k+2) g, r_{3 / 8}\right)+2(\bmod 8) .
$$

Also $\left(X_{2 k},(k+2) g, r_{-1 / 8}\right)$ and $\left(X_{2 k}, k g, r_{3 / 8}\right)$ are not proper.

If $C$ is a collection of disjoint ovals in $\mathbb{R P}(2)$, let $B^{+}(C)$ be the closed surface in $\mathbb{R P}(2)$ with boundary $C$ which is the closure of the set of points which lie inside an odd number of ovals of $C$. Let $B^{-}(C)$ be the closure of $\mathbb{R P}(2) \backslash B^{+}(C)$.

Proposition 4.3. Let $C$ be a collection of disjoint ovals in $\mathbb{R P}(2)$ with an even number of components. There is equivalence between:

(1) $(C, \gamma, r)$ is proper for some $\gamma$ and $r$.

(2) $(C, \gamma, r)$ is proper for all possible $\gamma$ (i.e., both 0 and $2 g$ ) and for all $r$.

(3) $C$ is odd.

(4) Every component of $B^{+}(C)$ has even Euler characteristic.

Proof. Each component $C_{i}$ represents $2 g \in H_{1}(\mathscr{T})$. Thus $2 r\left(\left[C_{i}\right]\right)=8 r[g]=1$ $(\bmod 2)$. As $C$ has an even number of components and each oval represents $2 g$, we have that $[C]=0 \in H_{1}(\mathscr{T})$. Thus $\gamma$ must be either 0 or $2 g$. Also $C$ is proper for either $\gamma$ and either $r$ if and only if $\operatorname{lk}\left(C_{i}, C \backslash C_{i}\right)$ is odd. On the other hand, $\operatorname{lk}\left(C_{i}, C \backslash C_{i}\right)$ is odd if and only if $C$ is odd. The equivalence of the last two conditions is easily seen.

The definitions of this paragraph are due to Rokhlin [1978]. A linked pair of ovals is called positive or negative according to whether the pair contributes to $\Pi^{+}$ or $\Pi^{-}$. An odd oval is called disoriented if forms a negative pair with the even oval that immediately surrounds it. Let $d$ denote the number of disoriented ovals. Let $D^{+}$denote the number of positive pairs with disoriented outer oval. Similarly let $D^{-}$denote the number of negative pairs with disoriented outer oval. Rokhlin observed that

$$
\Pi^{+}-\Pi^{-}=n-2\left(d-D^{+}+D^{-}\right) .
$$

If $C$ is odd, it is easy to see that $D^{+}+D^{-}$is even. Thus, if $C$ is odd, we have

$$
2 d \equiv \Pi^{+}-\Pi^{-}-n(\bmod 4) .
$$

Proposition 4.4. Let $C$ be an odd collection of disjoint ovals in $\mathbb{R P}(2)$. Then $\left(C,\left(\Pi^{+}-\Pi^{-}-p\right) g, r\right)$ and $\left(C,\left(\Pi^{+}-\Pi^{-}-p-2\right) g, r\right)$ are proper for either $r$. 
Moreover

$$
\begin{array}{rlrl}
\operatorname{Arf}\left(C,\left(\Pi^{+}-\Pi^{-}-p\right) g, r_{-1 / 8}\right) & \equiv \operatorname{Arf}\left(C,\left(\Pi^{+}-\Pi^{-}-p\right) g, r_{3 / 8}\right) & \\
& \equiv \Pi^{+}-\Pi^{-}-p & & (\bmod 8), \\
\operatorname{Arf}\left(C,\left(\Pi^{+}-\Pi^{-}-p-2\right) g, r_{3 / 8}\right) & \equiv \Pi^{+}-\Pi^{-}-p-2 & & (\bmod 8), \\
\operatorname{Arf}\left(C,\left(\Pi^{+}-\Pi^{-}-p+2\right) g, r_{-1 / 8}\right) & \equiv \Pi^{+}-\Pi^{-}-p+2 & & (\bmod 8) .
\end{array}
$$

Proof. By Proposition 4.3, every component of $B^{+}$has even Euler characteristic. Thus we can pick a vector field on $B^{+}$which is tangent to the boundary and pointed in the direction of the orientation of $C$ with $(n-p) / 2$ zeros of index -2 . This defines a surface in $\mathscr{T}$ lying over $B^{+}$with $(n-p) / 2$ disks removed around the zeros, which we denote by $z_{i}$. We complete this surface to form a spanning surface $F$ for $L(C)$ in $\mathcal{T}$ by adding Möbius bands above the disks around the zeros. We do this so that the cores of these Möbius bands, which we denote by $x_{i}$, are the fibers over $z_{i}$ of the map from $F$ to $\mathbb{R P}(2)$. This is similar to the construction of spanning surfaces in [Gilmer 1996, §1]. Then

$$
q_{r, F}\left(x_{i}\right) \equiv-1-4 r(2 g) \equiv 1(\bmod 4) .
$$

Since $B^{+}$is a planar surface,

$$
\beta\left(q_{r, F}\right) \equiv \frac{1}{2}(n-p)(\bmod 8) .
$$

We have

$$
\mu(F)=\frac{1}{2}\left(n+p-2\left(\Pi^{+}-\Pi^{-}\right)\right) .
$$

We have

$$
\gamma(F)=\left(\frac{1}{2}(n-p)+d\right) 2 g .
$$

Using (4-4), this becomes

$$
\gamma(F)=\left(\Pi^{+}-\Pi^{-}-p\right) g .
$$

Together with (3-1) gives the first equation. The last two equations follow from this and Proposition 4.1.

Proposition 4.5. Let $C$ be a collection of disjoint ovals in $\mathbb{R P}(2)$ with an odd number of components. There is equivalence between:

(1) $(C, \gamma, r)$ is proper for some $\gamma$, and $r$.

(2) $(C, \gamma, r)$ is proper for all possible $\gamma$ (that is, both $g$ and $-g$ ) and for all $r$.

(3) $C$ is even.

(4) Every component of $B^{-}(C)$ has even Euler characteristic. 
Proof. Since $C$ has an odd number of components and each oval represents $2 g$, we have $[C]=2 g \in H_{1}(\mathscr{T})$. Thus $\gamma$ must be either $g$ or $-g$. Also $C$ is proper for either $\gamma$ and either $r$ if and only if $\operatorname{lk}\left(C_{i}, C \backslash C_{i}\right)$ is even. On the other hand, $\operatorname{lk}\left(C_{i}, C \backslash C_{i}\right)$ is even if and only if $C$ is even. The equivalence of the last two conditions is easily seen.

Proposition 4.6. Let $C$ be an even collection of disjoint ovals in $\mathbb{R P}(2)$, then $(C, \pm g, r)$ are proper for both $r=r_{-1 / 8}$ and $r=r_{3 / 8}$. Moreover

$$
\begin{gathered}
\operatorname{Arf}\left(C, \pm g, r_{-1 / 8}\right) \equiv \Pi^{+}-\Pi^{-}-n+\frac{1}{2}(\bmod 8) \\
\operatorname{Arf}\left(C, \pm g, r_{3 / 8}\right) \equiv \Pi^{+}-\Pi^{-}-n-\frac{3}{2}(\bmod 8)
\end{gathered}
$$

Proof. By Proposition 4.5, every component of $B^{-}$has even Euler characteristic. Thus we can pick a vector field on $B^{-}$which is tangent to the boundary and pointed in the direction of the orientation of $C$ with $(p-n-1) / 2$ zeros of index -2 . This defines a surface in $\mathcal{T}$ lying over $B^{-}$with $(p-n-1) / 2$ disks removed around the zeros. As above, we complete this surface to form a spanning surface $F$ for $L(C)$ in $\mathcal{T}$ by adding Möbius bands above the removed disks around the zeros. $B^{-}$ is a planar surface disjoint union a Möbius band with some holes removed. The same can be said of the surface we obtain when we delete neighborhoods of the singularities of the vector field. One can see the $\gamma(F)$ is either $g$ or $-g$. As we will see, this allows us to compute our Arf invariants without ambiguity. Let us now write $\gamma(F)= \pm g$, and read plus, if indeed it is plus, and read minus, if indeed it is minus.

As in proof of Proposition 4.4, we let $x_{i}$ denote the cores of these Möbius bands. Equation (4-5) gives us $q_{r, F}\left(x_{i}\right)$. Let $\alpha$ denote an orientation reversing curve in $B^{-}$, then, as in (4-2) and (4-3),

$$
q_{r, F}(\alpha) \equiv \frac{1}{2}-4 r(g) \equiv\left\{\begin{array}{ll}
+1 & \text { if } r=r_{-1 / 8} \\
-1 & \text { if } r=r_{3 / 8}
\end{array}\right\} \quad(\bmod 4) .
$$

Thus

$$
\beta\left(q_{r, F}\right) \equiv \frac{1}{2}(p-n-1)+\left\{\begin{array}{ll}
+1 & \text { if } r=r_{-1 / 8}, \\
-1 & \text { if } r=r_{3 / 8},
\end{array}\right\} \quad(\bmod 8) .
$$

Equation (4-6) gives $\mu(F)$. Plugging this into (3-1), we obtain the stated results but where must read \pm according to whether $\gamma(F)$ is $\pm g$. However an application of Proposition 4.1 to both these equations shows that they must hold for the other choice of $\gamma$ as well.

\section{Main results}

By the proof of [Gilmer 1996, Theorem 3.1], we have: 
Theorem 5.1. If $A_{\mathbb{R}}$ is a nodal $M$-curve of degree $2 k$, then $\mathscr{T}\left(A_{\mathbb{R}}\right)$ is planar cobordant to $\mathscr{T}\left(X_{2 k}\right)$.

Corollary 5.2. Let $A_{\mathbb{R}}$ be a nodal M-curve of degree $2 k . \mathscr{T}\left(A_{\mathbb{R}}\right)$ is homologous to $2 \mathrm{~kg} \in H_{1}(\mathcal{T})$. If $k$ is even, $A_{\mathbb{R}}$ cannot be weakly equivalent to an even simple curve. If $k$ is odd, $A_{\mathbb{R}}$ cannot be weakly equivalent to an odd simple curve.

The next theorem follows from Proposition 4.2, the invariance of Arf invariants of proper links under planar cobordism, and Theorem 5.1.

Theorem 5.3. Let $A_{\mathbb{R}}$ be a nodal $M$-curve of degree $2 k$ and suppose $\left(A_{\mathbb{R}}, k g, r_{-1 / 8}\right)$ is proper. Then $\operatorname{Arf}\left(A_{\mathbb{R}}, k g, r_{-1 / 8}\right) \equiv \frac{1}{2} k^{2}(\bmod 8)$.

We can now give the proof of Theorem 1.1. Theorem 5.3 is more general but its application requires that one calculate $\operatorname{Arf}\left(A_{\mathbb{R}}, k g, r_{-1 / 8}\right)$.

Proof of Theorem 1.1. Suppose $k$ is even, and $A_{\mathbb{R}}$ is weakly equivalent to an odd simple curve. Then, by Proposition $4.3,\left(A_{\mathbb{R}}, k g, r_{-1 / 8}\right)$ is proper. So, by Theorem 5.3 ,

$$
\operatorname{Arf}\left(A_{\mathbb{B}}, 0, r_{-1 / 8}\right) \equiv \frac{1}{2} k^{2}(\bmod 8)
$$

By Proposition 4.4, one of $\left(\Pi^{+}-\Pi^{-}-p\right) g$ or $\left(\Pi^{+}-\Pi^{-}-p+2\right) g$ is zero, and $\operatorname{Arf}\left(C, 0, r_{-1 / 8}\right) \equiv \Pi^{+}-\Pi^{-}-p+ \begin{cases}0(\bmod 8) & \text { if } \Pi^{+}-\Pi^{-}-p \equiv 0(\bmod 4), \\ 2(\bmod 8) & \text { if } \Pi^{+}-\Pi^{-}-p \equiv 2(\bmod 4) .\end{cases}$

Since $\mathscr{T}(C)$ and $\mathscr{T}\left(A_{\mathbb{R}}\right)$ are planar cobordant, we have

$$
\operatorname{Arf}\left(A_{\mathbb{R}}, 0, r_{-1 / 8}\right) \equiv \operatorname{Arf}\left(C, 0, r_{-1 / 8}\right)(\bmod 8) .
$$

This gives the $k$ even case.

Now suppose $k$ is odd, and $A_{\mathbb{R}}$ is weakly equivalent to an even simple curve. Then, by Proposition $4.5,\left(A_{\mathbb{R}}, k g, r_{-1 / 8}\right)$ is proper. So, by Theorem 5.3,

$$
\operatorname{Arf}\left(A_{\mathbb{R}}, k g, r_{-1 / 8}\right) \equiv \frac{1}{2} k^{2}(\bmod 8) .
$$

By Proposition 4.6,

$$
\operatorname{Arf}\left(C, k g, r_{-1 / 8}\right) \equiv \Pi^{+}-\Pi^{-}-n+\frac{1}{2}(\bmod 8) .
$$

Since $\mathscr{T}(C)$ and $\mathscr{T}\left(A_{\mathbb{R}}\right)$ are planar cobordant, we have

$$
\operatorname{Arf}\left(A_{\mathbb{R}}, k g, r_{-1 / 8}\right) \equiv \operatorname{Arf}\left(C, k g, r_{-1 / 8}\right)(\bmod 8) .
$$

This gives the $k$ odd case.

We now show how Fiedler's original congruence for certain nonsingular curves is equivalent to Theorem 1.1 when $A_{\mathbb{R}}$ is a simple curve, even or odd, as the case may be. 
Theorem 5.4 (Fiedler). Let $A_{\mathbb{R}}$ be a nonsingular M-curve of degree $2 k$.

If $k$ is even, and $A_{\mathbb{R}}$ is an odd simple curve, then

$$
p-n \equiv-k^{2}(\bmod 16) \text {. }
$$

If $k$ is odd, and $A_{\mathbb{R}}$ is an even simple curve, then

$$
p-n \equiv 1(\bmod 16) \text {. }
$$

Proof. We have Gudkov's congruence:

$$
p-n \equiv k^{2}(\bmod 8) .
$$

Harnack's inequality is extremal:

$$
p+n=1+\left(\begin{array}{c}
2 k-1 \\
2
\end{array}\right)=2 k^{2}-3 k+2 .
$$

Adding Equations (5-1) and (5-2), and dividing by 2:

$$
p \equiv \frac{3 k^{2}-3 k+2}{2}(\bmod 4) \text {. }
$$

According to [Rokhlin 1978, Equation 4],

$$
\Pi^{+}-\Pi^{-}=\frac{(k-1)(k-2)}{2}=\frac{k^{2}-3 k+2}{2} .
$$

Subtracting (5-3) from (5-4), we obtain

$$
\Pi^{+}-\Pi^{-}-p \equiv-k^{2}(\bmod 4) .
$$

At this point, we consider separately three different cases: $k \equiv 0(\bmod 4), k \equiv 2$ $(\bmod 4)$, and $k \equiv 1(\bmod 2)$.

Now assume that $k \equiv 0(\bmod 4)$ and $A_{\mathbb{R}}$ is an odd simple curve. By (5-5), $\Pi^{+}-\Pi^{-}-p \equiv 0(\bmod 4)$. Thus by Theorem 1.1

$$
\Pi^{+}-\Pi^{-}-p \equiv \frac{1}{2} k^{2} \equiv 0(\bmod 8) .
$$

Thus, using (5-4),

$$
2 p=k^{2}-3 k+2 \equiv-3 k+2(\bmod 16) .
$$

Subtracting $(5-2), p-n=-2 k^{2} \equiv 0(\bmod 16)$, which agrees with the desired conclusion.

Now assume that $k \equiv 2(\bmod 4)$ and $A_{\mathbb{R}}$ is an odd simple curve. We still have $\Pi^{+}-\Pi^{-}-p \equiv 0(\bmod 4)$, but now $\frac{1}{2} k^{2} \equiv 2 \bmod 8$. So, by Theorem 1.1,

$$
\Pi^{+}-\Pi^{-}-p \equiv \frac{1}{2} k^{2}-2 \equiv 0(\bmod 8) .
$$


Using Equations (5-4) and (5-2), as above, we obtain the conclusion of the theorem to be proved.

Now assume $k \equiv 1(\bmod 2)$ and $A_{\mathbb{R}}$ is an even simple curve. By Theorem 1.1,

$$
\Pi^{+}-\Pi^{-}-n \equiv \frac{1}{2}\left(k^{2}-1\right) \equiv 0(\bmod 8) .
$$

Using (5-4) we get $-2 n \equiv 3 k-3(\bmod 16)$, and finally, using (5-2),

$$
p-n \equiv 2 k^{2}-1 \equiv 1(\bmod 16) \text {. }
$$

\section{Fiedler's curves}

6.1. Prohibiting a curve of degree 6. Consider the hypothetical curve of degree six prohibited by Fiedler [1986, Figure 1]. We denote this $\mathscr{I}$-curve by $C_{1}$. We note that there is only one orientation on this two component curve up isotopy. So we equip $C_{1}$ with the orientation which allows the unsmoothing move that we take below. $C_{1}$ is proper for $\gamma= \pm g$ and $r$ equal either $r_{-1 / 8}$ or $r_{3 / 8}$. We perform some safe type II moves and type III moves on $C_{1}$ followed by an unsmoothing move, and a balanced type I move to obtain this $\mathscr{I}$-curve:

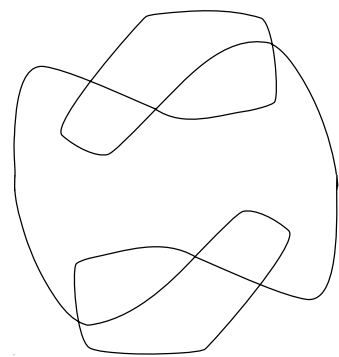

which we denote by $C_{2}$. Since the unsmoothing move decreases the number of components, $\left(C_{2}, \pm g, r\right)$ must be proper, [Gilmer 1993a, Corollary 6.10]. Thus

$$
\operatorname{Arf}\left(C_{1}, \pm g, r\right) \equiv \operatorname{Arf}\left(C_{2}, \pm g, r\right)(\bmod 8)
$$

for either $r$.

We pick a "vector field" on $B^{-}\left(C_{2}\right)$ which is tangential to the boundary pointed in the direction of the orientation, has a whole arc of tangent directions at each double point (as in the spanning surface for td in section 3 ) and has a single singularity of index -2 . This describes a spanning surface $F$ for $L\left(C_{2}\right)$. We calculate that $\gamma(F)=-g$ and $\mu(F)=\frac{-9}{2}$. We have a basis for $H_{1}(\bar{F}, \mathbb{Z} / 2 \mathbb{Z})$ consisting of two 1-sided curves $\delta_{1}, \delta_{2}$ on $F$ in the affine part of picture, the fiber $x$ over the singularity of index -2 , and the line at infinity $\alpha$. As in (4-1), (4-5), and (4-7) we have respectively

$$
q_{-1 / 8, F}\left(\delta_{1}\right)=q_{r_{-1 / 8}, F}\left(\delta_{2}\right)=1, \quad q_{r_{-1 / 8}, K}(x)=1, \quad q_{r_{-1 / 8}, K}(\alpha)=1 .
$$


Thus $\beta\left(q_{r_{-1 / 8}, F}\right) \equiv 4(\bmod 8)$, and hence

$$
\operatorname{Arf}\left(C_{2}, 3 g, r_{-1 / 8}\right) \equiv \operatorname{Arf}\left(C_{2},-g, r_{-1 / 8}\right) \equiv 4+\frac{9}{2} \equiv \frac{1}{2} \not \equiv \frac{9}{2}(\bmod 8) .
$$

By Theorem 5.3, $C_{1}$ is not a real algebraic curve of degree 6.

6.2. Prohibiting a curve of degree 8 . Consider the hypothetical curve of degree eight prohibited by Fiedler [1986, Figure 2]. We denote this $\mathscr{I}$-curve by $C_{3}$ and equip it with the only semiorientation on $C_{3}$, up to isotopy, which is consistent with [Rokhlin 1978, Equation 4] and Fiedler's alternation of ovals with respect to a pencil of lines [1982] when applied to $C_{3}$ smoothed. For this calculation, we use the language of floppy curves as developed in [Gilmer 1996]. We draw a real floppy curve $C_{4}$ in $\mathbb{R P}(2)$ whose corresponding link in $\mathcal{T}$ is isotopic to the link that corresponds to $C_{3}$ with this orientation:

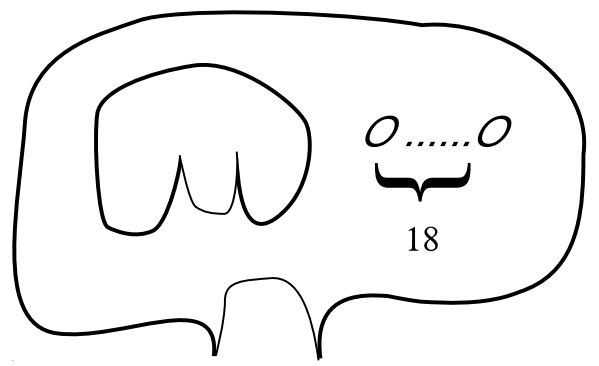

The outer curve with two flops is oriented clockwise. The inner curve with two flops is oriented counterclockwise. Ten of the ovals are oriented counterclockwise and eight clockwise.

We check that $C_{3}$ is proper for $\gamma=0$ and $r=r_{-1 / 8}$. We can extend the vector field on the boundary over $B^{+}$with ten singularities of index -2 . This specifies a spanning surface $F$. One calculates that $\gamma(F)=0$ and $\mu(F)=6$. Using Equation (4-5), we thus get $\beta\left(q_{r_{-1 / 8}, F}\right) \equiv 10 \equiv 2(\bmod 8)$. Therefore

$$
\operatorname{Arf}\left(C_{3}, 0, r_{-1 / 8}\right) \equiv 2-6 \equiv 4 \not \equiv 0(\bmod 8) .
$$

By Theorem 5.3, $C_{3}$ is not a real algebraic curve of degree 8 .

\section{References}

[Fiedler 1982] T. Fidler, "Pencils of lines and the topology of real algebraic curves", Izv. Akad. Nauk SSSR Ser. Mat. 46:4 (1982), 853-863. In Russian; translated in Math. USSR Izv. 21 (1983), 161-170. MR 84e:14019 Zbl 0522.14014

[Fiedler 1983] T. Fidler, "New congruences in the topology of real plane algebraic curves", Dokl. Akad. Nauk SSSR 270:1 (1983), 56-58. In Russian; translated in Sov. Math. Dokl. 27 (1983), 566568. MR 85b:14039 Zbl 0541.14022 
[Fiedler 1986] T. Fidler, "New congruences in the topology of singular real algebraic plane curves", Dokl. Akad. Nauk SSSR 286:5 (1986), 1075-1079. In Russian; translated in Sov. Math. Dokl. 33 (1986), 262-266. MR 87j:14053 Zbl 0608.14025

[Finashin 1996] S. M. Finashin, "On the topology of real plane algebraic curves with nondegenerate quadratic singularities", Algebra i Analiz 8:6 (1996), 186-204. In Russian; translated in St. Petersburg Math. J. 8:6 (1997) 1039-1051. MR 99c:14067 Zbl 0885.14030

[Gilmer 1992] P. Gilmer, "Real algebraic curves and link cobordism", Pacific J. Math. 153:1 (1992), 31-69. MR 93c:57005 Zbl 0784.57002

[Gilmer 1993a] P. M. Gilmer, "Link cobordism in rational homology 3-spheres", J. Knot Theory Ramifications 2:3 (1993), 285-320. MR 94m:57012 Zbl 0797.57003

[Gilmer 1993b] P. Gilmer, "A method for computing the Arf invariants of links", pp. 174-181 in Quantum topology, Ser. Knots Everything 3, World Sci., River Edge, NJ, 1993. MR 95d:57003 Zbl 0839.57005

[Gilmer 1996] P. Gilmer, "Real algebraic curves and link cobordism. II", pp. 73-84 in Topology of real algebraic varieties and related topics, edited by V. Kharlamov et al., Amer. Math. Soc. Transl. Ser. 2 173, Amer. Math. Soc., Providence, RI, 1996. MR 97e:57006 Zbl 0864.57008

[Gilmer 2000] P. M. Gilmer, "Floppy curves, with applications to real algebraic curves", pp. 39-76 in Real algebraic geometry and ordered structures (Baton Rouge, LA, 1996), edited by C. Delzell and J. Madden, Contemp. Math. 253, Amer. Math. Soc., Providence, RI, 2000. MR 2001j:14079 Zbl 0986.14039

[Gordon and Litherland 1978] C. M. Gordon and R. A. Litherland, "On the signature of a link", Invent. Math. 47:1 (1978), 53-69. MR 58 \#18407 Zbl 0391.57004

[Kharlamov and Viro 1988] V. M. Kharlamov and O. Y. Viro, "Extensions of the Gudkov-Rohlin congruence", pp. 357-406 in Topology and geometry-Rohlin Seminar, edited by O. Y. Viro, Lecture Notes in Math. 1346, Springer, Berlin, 1988. MR 90a:14030 Zbl 0678.14004

[Rokhlin 1978] V. A. Rokhlin, "Complex topological characteristics of real algebraic curves", Uspekhi Mat. Nauk 33:5 (1978), 77-89. In Russian; translated in Russian Math. Surveys 33:5 (1978), 85-98. MR 81m:14024 Zbl 0437.14013

[Viro 1978] O. Y. Viro, “Обобщенные неравенства Петровского и Арнольда на кривых с особенностями”, Uspekhi Mat. Nauk 33:4 (1978), 145-146.

[Viro 1986] O. Y. Viro, "Progress in the topology of real algebraic varieties over the last six years", Uspekhi Mat. Nauk 41:3 (1986), 45-67. In Russian; translated in Russian Math. Surveys 41:3 (1986), 55-82. MR 87m:14023 Zbl 0619.14015

[Viro and Orevkov 2001] O. Y. Viro and S. Y. Orevkov, "Congruence modulo 8 for real algebraic curves of degree 9", Uspekhi Mat. Nauk 56:4 (2001), 137-138. In Russian; translated in Russian Math. Surveys 56:4 (2001), 770-771. MR 2002h:14097 Zbl 1049.14045

Received October 5, 2005.

\section{PATRICK M. GILMER}

DEPARTMENT OF MATHEMATICS

LOUISIANA STATE UNIVERSITY

BATON ROUGE, LA 70803

UNITED STATES

gilmer@math.lsu.edu

www.math.lsu.edu/ gilmer/ 